05,02

\title{
Сравнительный анализ магнитосиловых характеристик магнитных линеек на основе постоянных магнитов и сверхпроводящих лент
}

\author{
(С) А.И. Подливаев ${ }^{1,2}$, И.А. Руднев ${ }^{1,3}$ \\ ${ }^{1}$ Национальный исследовательский ядерный университет „МИФИ“, \\ Москва, Россия \\ ${ }^{2}$ Научно-исследовательский институт проблем развития научно-образовательного потенциала молодежи, \\ Москва, Россия \\ ${ }^{3}$ Научно-технический университет „Сириус“, \\ Сочи, Россия \\ E-mail: AIPodlivayev@mephi.ru
}

Поступила в Редакцию 28 апреля 2021 г.

В окончательной редакции 28 апреля 2021 r.

Принята к публикации 11 мая 2021 г.

Теоретически рассмотрены характеристики магнитолевитационных систем двух типов, отличающихся по способу создания градиентного магнитного поля. В первом случае градиентное магнитное поле в системе источник магнитного поля-сверхпроводник создается с помощью магнитной линейки (набора) из постоянных $\mathrm{NdFeB-магнитов.} \mathrm{Во} \mathrm{втором} \mathrm{случае} \mathrm{магнитная} \mathrm{линейка} \mathrm{представляет} \mathrm{собой} \mathrm{набор} \mathrm{лент} \mathrm{высокотемпературных}$ сверхпроводников, намагниченных внешним полем и выполняющих роль источника градиентного поля. Проведенные расчеты показали, что при температуре жидкого азота магнитные линейки на основе пакетов ВТСП-лент уступают по эффективности наборам из постоянных магнитов при размерах периодов магнитной линейки до $38 \mathrm{~mm}$. При большей величине периода магнитные линейки на основе пакетов ВТСП-лент более эффективны. Также показано, что эффективность магнитных линеек на основе пакетов ВТСП-лент резко возрастает с понижением температуры.

Ключевые слова: сверхпроводник, критический ток, $\mathrm{GdBaCuO}$, магнитолевитационные системы.

DOI: 10.21883/FTT.2021.10.51398.100

\section{1. Введение}

Одним из базовых элементов различных бесконтактных устройств, функционирующих на основе магнитной левитации (левитационный транспорт, бесконтактные подшипники и т.п.) является комбинация магнитов, создающих неоднородное по пространству магнитное поле [1-6]. Необходимая градиентная конфигурация магнитного поля может быть создана как с помощью постоянных магнитов (ПМ), так и посредством намагниченных во внешнем поле сверхпроводников второго рода (СП). При этом СП в силу явления пиннинга могут захватывать магнитный поток, и долгое время сохранять на своей поверхности огромные, по сравнению с постоянными магнитами величины магнитной индукции (см., например, [7]). Соответственно, в первом случае силовое взаимодействие возникает в системе сверхпроводникпостоянный магнит (это наиболее распространенный тип магнитолевитационных систем). Во втором случае сверхпроводник взаимодействует с градиентным полем другого намагниченного сверхпроводника. Преимущество системы СП-ПМ в том, что магнитная часть устройства на основе постоянного магнита не нуждается в охлаждении, в то время как в паре СП-СП при недостаточном охлаждении в намагниченном сверхпро- воднике возможно возникновение термодинамической нестабильности (см., например, [8,9]), которая может приводить к спонтанной потери остаточного магнитного момента.

В последующем тексте настоящей работы источник неоднородного магнитного поля (либо на основе постоянных магнитов, либо сверхпроводников) будет обозначаться как магнитная линейка, а вторая часть магнитосиловой системы - верхний сверхпроводящий слой (ВСС). В отличие от сверхпроводника в магнитной линейке, ВСС исходно не намагничен. Далее будет показано, что его магнитолевитационные характеристики существенно зависят от типа магнитной линейки. СП магнитная линейка может быть создана на основе объемных сверхпроводников, однако более технологичным подходом является применение пакетов ВТСПлент, которые не уступают по своим характеристикам объемным сверхпроводникам [10]. В настоящей работе сопоставляется численно определенная эффективность одномерной магнитной линейки, собранной из постоянных магнитов $\mathrm{NdFeB}$ с эффективностью линейки, созданной на основе стопок из ленточных композитных высокотемпературных сверхпроводников (ВТСП) $\mathrm{GdBa}_{2} \mathrm{Cu}_{3} \mathrm{O}_{7-x}$. Мерой эффективности в настоящей работе выбрана сила взаимодействия ВСС и 
магнитных линеек различных типов, но обладающих одинаковыми геометрическими размерами. Предполагается, что ВТСП-лента функционирует при трех рабочих температурах: температуре кипения жидкого азота $(77 \mathrm{~K})$, жидкого азота при пониженном давлении $(65 \mathrm{~K})$ и при $30 \mathrm{~K}$ (температура кипения жидкого неона $27 \mathrm{~K})$.

В настоящей работе нас интересуют неоднородные конфигурации токов и соответствующих им магнитных полей ВТСП-лент. Неоднородные состояния магнитных пленок различного типа изучаются в рамках различных подходов в зависимости от масштаба неоднородности. При теоретическом исследовании на атомном уровне может применяться теория функционала плотности [11]. Неоднородность обусловленная микроскопической зернистой структурой может приводить к необычным физическим явлениям, например, появлению фазовых переходов по току, аналогичных наблюдаемым в гранулированных сверхпроводниках $\mathrm{YBa}_{2} \mathrm{Cu}_{3} \mathrm{O}_{7-x}$ [12]. В работах $[13,14]$ экспериментально показано влияние грануляции структуры ВТСП на гистерезисный характер магнитосопротивления. Для левитационных приложений характерен интервал размеров неоднородностей, превышающий несколько миллиметров. Именно такой характерный размер неоднородностей магнитного поля и токов будет рассмотрен в настоящей статье.

\section{2. Характеристики градиентного магнитного поля для двух типов магнитных линеек}

\section{1. Магнитная линейка на основе постоянных магнитов}

Структура интересующей нас магнитной линейки (МЛ) представлена на рис. $1, a$. Элементом МЛ является полоса намагниченного соединения $\mathrm{NdFeB}$. В системе декартовых координат $(X, Y, Z)$ полоса имеет неограниченную длину $(-\infty<Y<+\infty)$. Высота полосы равна $Z_{h}\left(-Z_{h}<Z<0\right)$, а ширина (в направлении $\left.X\right)-$ $a / 2$. Магнитные полосы расположены в направлении оси $X$ так, что соседние полосы имеют противоположную намагниченность. Направление плотности магнитного момента полос представлено на рис. 1, $a$ жирными стрелками. В дальнейшем предполагается, что эта периодическая структура не ограничена в направлении оси $X$. Период структуры равен $a$. Магнитное поле над МЛ также периодично. На рис. 1, $a$ схематически представлена величина $B_{Z}-$ вертикальная компонента индукции магнитного поля при $Z=0$. При удалении от поверхности МЛ магнитное поле затухает значительно быстрее, чем аналогичная величина для отдельной магнитной полосы. Действительно: допустим, что магнитная полоса намагничена однородно. Вектор плотности магнитного момента имеет следующие компоненты:
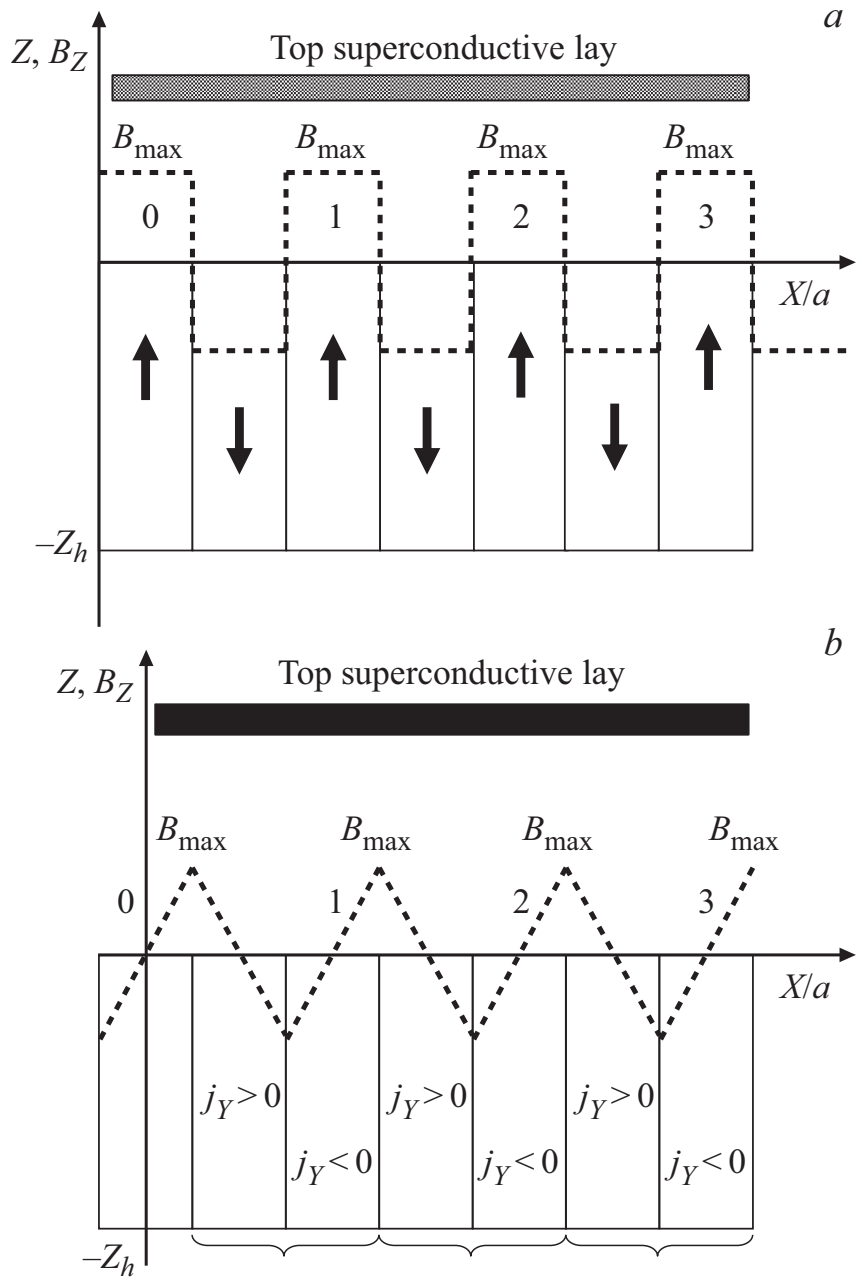

Рис. 1. Сечение магнитных линеек на основе постоянных магнитов $(a)$ и пакетов сверхпроводящих лент $(b)$. Пунктирными линиями схематически изображена величина нормальной компоненты индукции магнитного поля на поверхности магнитных линеек. Над магнитными линейками расположен ВСС - левитирующий сверхпроводящий слой (обозначен заштрихованным прямоугольником малой толщины).

$g_{X}=g_{Y}=0, g_{Z} \neq 0$. Связь плотности магнитного момента $\mathbf{g}(\mathbf{r})$ (в точке с радиус-вектором $\mathbf{r}$ ) распределенной в области $S$ и вектором магнитной индукции $\mathbf{B}\left(\mathbf{R}_{0}\right)$ определяется следующим выражением [15]:

$\mathbf{B}\left(\mathbf{R}_{0}\right)=\frac{\mu_{0}}{4 \pi} \int_{S}\left[3 \frac{\left(\mathbf{g}(\mathbf{r})\left(\mathbf{R}_{0}-\mathbf{r}\right)\right)\left(\mathbf{R}_{0}-\mathbf{r}\right)}{\left|\mathbf{R}_{0}-\mathbf{r}\right|^{5}}-\frac{\mathbf{g}(\mathbf{r})}{\left|\mathbf{R}_{0}-\mathbf{r}\right|^{3}}\right] d^{3} r$,

где $\mu_{0}-$ магнитная проницаемость вакуума. Для приложений магнитной левитации в данной работе нас в первую очередь интересует вертикальная компонента магнитного моля над МЛ. Интеграл (1) для отдельной магнитной полосы, которая расположена в области $\left(0 \leq X \leq a / 2 ;-\infty<Y<+\infty\right.$; $\left.-Z_{h} \leq Z \leq 0\right)$ дает следующее выражение для вертикальной компоненты 


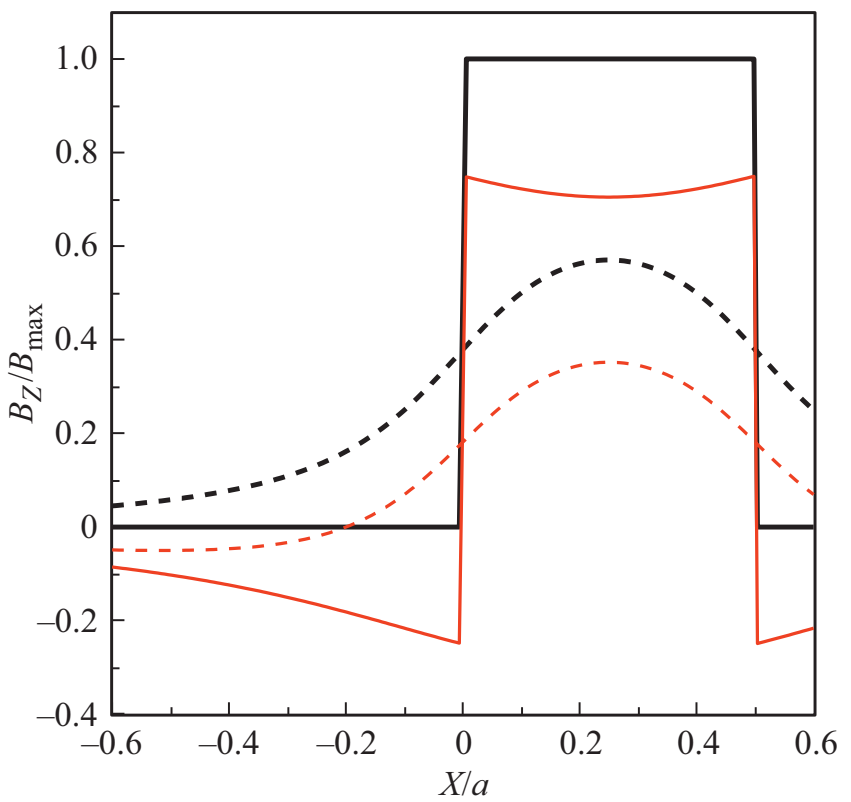

Рис. 2. Вертикальная компонента $B_{Z}$ индукции магнитного поля над отдельной магнитной полосой. Жирная черная сплошная линия $-Z=0, Z_{h}=\infty$. Жирная черная пунктирная линия $-Z=a / 5, Z_{h}=\infty$. Тонкая красная сплошная линия $Z=0, Z_{h}=a / 2$. Тонкая красная пунктирная линия $Z=a / 5$, $Z_{h}=a / 2$.

индукции магнитного поля над полосой $(0 \leq Z<+\infty)$

$$
\begin{aligned}
B_{Z}= & \frac{B_{\max }}{\pi}\left[\operatorname{arctg}\left(\frac{a / 2-X}{Z}\right)-\operatorname{arctg}\left(\frac{-X}{Z}\right)\right. \\
& \left.-\operatorname{arctg}\left(\frac{a / 2-X}{Z+Z_{h}}\right)+\operatorname{arctg}\left(\frac{-X}{Z+Z_{h}}\right)\right],
\end{aligned}
$$

где $B_{\max }$ - максимально возможная величина индукции магнитного поля при данной плотности магнитного момента.

На рис. 2 представлена зависимость $B_{Z}(X)$ при различных значениях $Z$ для полубесконечной в вертикальном направлении магнитной полосы $\left(Z_{h}=\infty\right)$ и для полосы, ширина и высота которой совпадают $\left(Z_{h}=a / 2\right)$. На этом рисунке видно, что магнитное поле полубесконечной в вертикальном направлении магнитной полосы заметно отличается от поля полосы, ширина и высота которой совпадают. В периодической магнитной линейке зависимость поля от высоты магнита существенно отличается от аналогичной зависимости в отдельной магнитной полосе.

Исходя из выражения (1) для периодической МЛ, вертикальная компонента $B_{Z}$ индукции магнитного поля может быть представлена в виде ряда Фурье следующим образом:

$$
\begin{aligned}
B_{Z}= & B_{\max } \sum_{n=0,1,2,3, \ldots} B_{n} \cdot \sin \left(\varphi_{n} X\right) \cdot \exp \left(-\varphi_{n} Z\right) \\
& \times\left(1-\exp \left(-\varphi_{n} Z_{h}\right)\right), \quad \varphi_{n}=2 \pi(2 n+1) / a .
\end{aligned}
$$

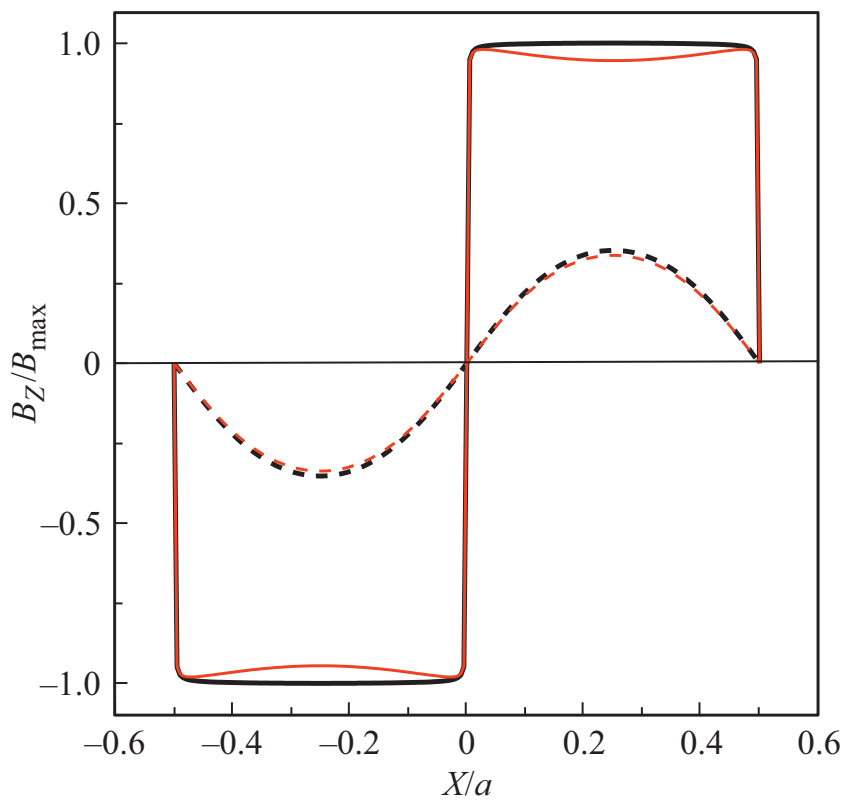

Рис. 3. Один период вертикальной компоненты $B_{Z}$ индукции магнитного поля над магнитной линейкой. Жирная черная сплошная линия $-Z=0, Z_{h}=\infty$. Жирная черная пунктирная линия $-Z=a / 5, Z_{h}=\infty$. Тонкая красная сплошная линия $Z=0, Z_{h}=a / 2$. Тонкая красная пунктирная линия $-Z=a / 5$, $Z_{h}=a / 2$

Коэффициенты Фурье Вn имеют следующий вид:

$$
B_{n}=4 /[\pi(2 n+1)] .
$$

На рис. 3 представлена зависимость $B_{Z}(X)$ при различных значениях $Z$ для полубесконечной в вертикальном направлении периодической магнитной линейки $\left(Z_{h}=\infty\right)$ и для МЛ, высота которой равна половине периода $Z_{h}=a / 2$. Сопоставление рис. 2 и 3 показывает, что в отличие от отдельной полосы, магнитное поле МЛ с высотой, которой равна половине периода $Z_{h}=a / 2$ практически не отличается от поля МЛ для $Z_{h}=\infty$.

\section{2. Магнитная линейка на основе пакетов ВТСП лент}

\subsection{1. Расчет распределения поля в рамках модели Бина}

Периодическая структура магнитной линейки может быть создана комбинацией пакетов ВТСП-лент. Градиентное магнитное поле при этом может создаваться двумя различными способами. Первый способ подразумевает пропускание через пакеты в направлении оси $Y$ транспортного тока. Аналогом стопки лент можно считать также короткозамкнутые катушки, изготовленные из ВТСП-лент [16]. Высота и ширина пакета равны, соответственно $a / 2$ и $Z_{h}$ (смотри рис. $\left.1, b\right)$. Плотность тока $j_{Y}$ в пакете не превышает критическую величину $j_{c}$. При втором способе возможно формирование необходимого профиля магнитного поля за счет остаточной 
намагниченности пакета (см., например обзор [17] и ссылки в нем, а также использование принципа „магнитного насоса“ $[18])$. Плотность тока в пакете во втором случае также ограничена величиной $j_{c}$. При втором способе ширина лент, образующих МЛ равна $a-$ в два раза больше, чем в обмотке с транспортным током. На рис. $1, b$ пакеты этих ВТСП-лент обозначены нижними фигурными скобками. При намагничивании пакетов ВТСП внешним магнитным полем лента намагничивается так, что наведенный ток в центре ленты равен нулю, а слева и справа от центральной линии наведенные токи имеют противоположный знак $[19,20]$. При этом, в случае высокой амплитуды индуцирующего поля практически вся лента находится в критическом состоянии. Ширина центральной части, в которой амплитуда наведенного тока ниже критической, мала по сравнению с шириной ленты $a$. Именно такой предельный случай рассматривается ниже при расчете МЛ на основе ВТСПлент.

В приближении модели Бина $[21,22]$, в которой пренебрегается зависимостью критического тока от магнитного поля, магнитное поле линейки на основе пакетов ВТСП-лент может быть вычислено аналитически. Связь токов с плотностью $\mathbf{j}(\mathbf{r})$ распределенных в области $S$ и магнитным полем $\mathbf{B}\left(\mathbf{R}_{0}\right)$ определяется следующим уравнением Био-Савара-Лапласа

$$
\mathbf{B}\left(\mathbf{R}_{0}\right)=\frac{\mu_{0}}{4 \pi} \int_{S} \frac{\mathbf{J}(\mathbf{r}) \times\left(\mathbf{R}_{0}-\mathbf{r}\right)}{\left|\mathbf{R}_{0}-\mathbf{r}\right|^{3}} d^{3} r .
$$

Исходя из выражения (5) для периодической МЛ, вертикальная компонента $B_{Z}$ индукции магнитного поля над линейкой может быть также представлена в виде ряда Фурье (3). В этом случае величина $B_{\max }$ и коэффициенты $B_{n}$ ряда Фурье определяются следующим выражением:

$$
\begin{gathered}
B_{\max }=\mu_{0} j_{c} a / 4, \quad B_{N}=2 \cdot \sin (q) / q^{2}, \\
q=\pi(2 n+1) / 2 .
\end{gathered}
$$

На рис. 4 представлена зависимость $B_{Z}(X)$ при различных значениях $Z$ для полубесконечной в вертикальном направлении периодической линейки на основе ВТСПлент с высотами $Z_{h}=\infty$ и $Z_{h}=a / 2$. На этом рисунке видно, что магнитное поле МЛ с высотой, которая равна половине периода $Z_{h}=a / 2$ практически не отличается от поля МЛ для $Z_{h}=\infty$. Для оценки эффективности МЛ в дальнейшем рассматриваем полубесконечную в вертикальном направлении линейку $\left(Z_{h}=\infty\right)$.

Магнитное поле МЛ быстро уменьшается при удалении от поверхности МЛ независимо от того, на основе каких элементов эта линейка собрана. При $Z>a$ преобладающей является Фурье-компонента с $n=0$ (смотри выражение (3)). Обозначим переменной $B_{p m}$ величину $B_{\max }$ для МЛ на основе постоянных магнитов. Для этой линейки с учетом выражений (3), (4) на больших

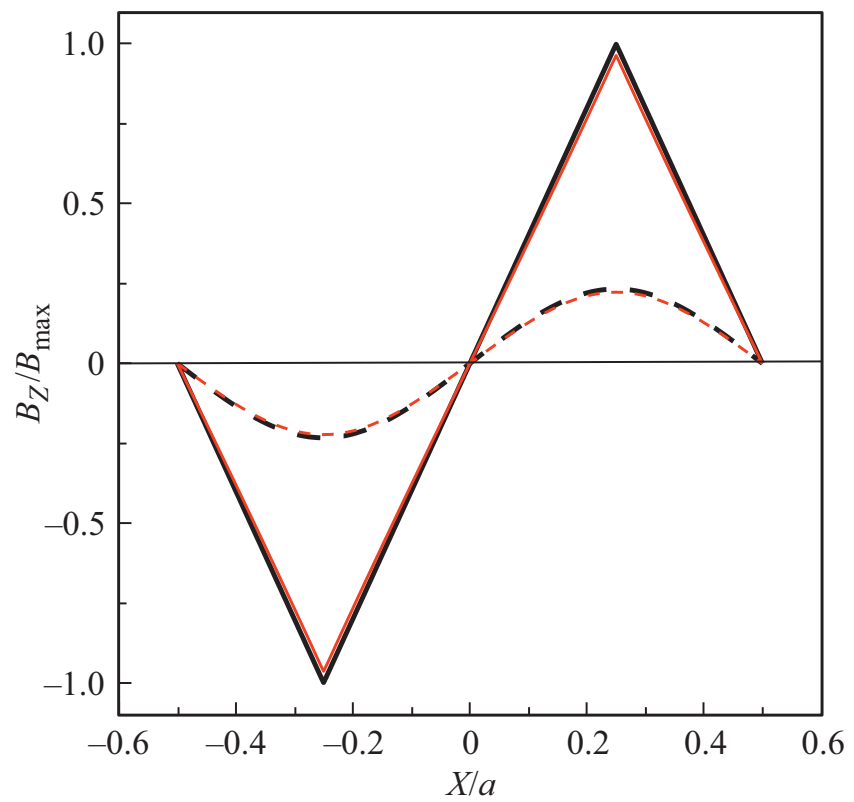

Рис. 4. Один период вертикальной компоненты $B_{Z}$ индукции магнитного поля над линейкой на основе пакетов ВТСП-лент. Черная жирная сплошная линия $-Z=a / 5, Z_{h}=\infty$. Тонкая красная сплошная линия $-Z=0, Z_{h}=a / 2$. Тонкая красная пунктирная линия $Z=a / 5, Z_{h}=a / 2$.

расстояниях от поверхности МЛ вертикальная компонента $B_{Z}$ индукции магнитного поля имеет следующий вид:

$$
B_{Z}=(4 / \pi) \cdot B_{p m} \cdot \sin (2 \pi X / a) \cdot \exp (-2 \pi Z / a) .
$$

Для МЛ на основе пакетов ВТСП-лент для предела $Z \gg a$ из выражений (3), (6) получаем следующую координатную зависимость индукции магнитного поля

$$
B_{Z}=\left(1 / \pi^{2}\right) \cdot \mu_{0} j_{c} \cdot a \cdot \sin (2 \pi X / a) \cdot \exp (-2 \pi Z / a) .
$$

При малой величине периода более эффективными являются МЛ на основе постоянных магнитов, а при больших значениях $a-$ более эффективными являются МЛ на основе пакетов ВТСП-лент. Определим граничный размер периода линейки $a_{b o u n d}$ при котором эффективности этих структур совпадают. Из выражений (7) и (8) получаем

$$
a_{\text {bound }}=4 \pi \cdot B_{p m} /\left(\mu_{0} j_{c}\right) .
$$

Для постоянных магнитов $\mathrm{NdFeB}$ полагаем величину $B_{p m}=0.5$ Т. При выборе ВТСП-ленты мы ориентировались на достаточно перспективную продукцию фирмы SuperOx (см., например, [23]). Для ВТСП-лент, произведенных фирмой SuperOx, в соответствие с данными работ $[24,25]$ плотность критического тока $J_{c}$ в чистом сверхпроводнике $\mathrm{GdBa}_{2} \mathrm{Cu}_{3} \mathrm{O}_{7-x}$ при температуре $T=77 \mathrm{~K}$ равна $2.77 \cdot 10^{10} \mathrm{~A} / \mathrm{m}^{2}$. Также как в работе [26] полагаем, что толщина ВТСП-слоя в 
ленте $h=1.5 \mu \mathrm{m}$. Полная толщина ленты $d=0.1 \mathrm{~mm}$ (основную часть составляют защитные слои меди и серебра, а также подложка из сплава хастеллой [25]). В этом случае эффективная объемная плотность критического тока $j_{c}=J_{c} \cdot h / d=4.155 \cdot 10^{8} \mathrm{~A} / \mathrm{m}^{2}$. При этом плотность поверхностного тока одной ленты $j_{0}=J_{c} \cdot h=4.155 \cdot 10^{4} \mathrm{~A} / \mathrm{m}$. Выражение (9) в этом случае дает граничное значение $a_{\text {bound }}=12 \mathrm{~mm}$. Из данных, приведенных в работе [24] для более низких температур 65 и $30 \mathrm{~K}$ следует $J_{c}\left(T=65 \mathrm{~K}=2 J_{c}(T=77 \mathrm{~K})\right.$ и $J_{c}(T=30 \mathrm{~K})=6 J_{c}(T=77 \mathrm{~K})$. Величины граничного значения $a_{\text {bound }}$ для этих температур равны соответственно 6 и $2 \mathrm{~mm}$. Однако при выводе формулы (9), не учитывалось подавляющее влияние собственного магнитного поля пакета ВТСП-лент на плотность критического тока.

\subsection{2. Расчет распределения поля в рамках двухэкспоненциальной модели}

В рамках двухэкспоненциального приближения [27] зависимость критического тока $j_{c}$ от величины индукции магнитного поля $B$ при температуре $T=77 \mathrm{~K}$ определяется следующим выражением:

$$
j_{c}(B)=A_{1} \cdot \exp \left(-|B| / \beta_{1}\right)+A_{2} \cdot \exp \left(-|B| / \beta_{2}\right) .
$$

На примере ВТСП-лент производства фирмы SuperOx, в работе [26] было показано, что это приближение точнее описывает экспериментальную зависимость $j_{c}(B)$, чем модель Кима [28,29], линейная модель [30], а также некоторые менее распространенные модели критического состояния сверхпроводников (см., например, обзор [31] и ссылки на литературу в нем), а в работе [32] показана адекватность данного приближения при описании ВТСП-пленок разных производителей при различных температурах.

Параметры приближения $A_{1}=2.3665 \cdot 10^{8} \mathrm{~A} / \mathrm{m}^{2}, A_{2}=$ $=1.7884 \cdot 10^{8} \mathrm{~A} / \mathrm{m}^{2}, \beta_{1}=0.1175$ Т и $\beta_{2}=1.2238 \mathrm{~T}$ определены в работе [26] подгонкой к экспериментальной зависимости работы [24] методом наименьших квадратов. В рассматриваемой геометрии МЛ $|B|=\sqrt{B_{X}^{2}+B_{Z}^{2}}$. Применение выражения (10) дает значение граничной величины $a_{\text {bound }}(T=77 \mathrm{~K})=38 \mathrm{~mm}$, что существенно превышает аналогичную величину в приближении Бина $(12 \mathrm{~mm})$. Из-за отсутствия детальных экспериментальных данных о полевой зависимости плотности критического тока при $T=65 \mathrm{~K}$ и $T=30 \mathrm{~K}$ для лент фирмы SuperOx $\mathrm{GdBa}_{2} \mathrm{Cu}_{3} \mathrm{O}_{7-x}$ приближенно считаем, что

$$
j_{c}(B, T)=\gamma(T) \cdot j_{c}(B, T=77 \mathrm{~K}) .
$$

Для соответствия экспериментальным данным работы [24] $\gamma(T=77 \mathrm{~K})=1, \gamma(T=65 \mathrm{~K})=2, \gamma(T=30 \mathrm{~K})=6$. Введем новые переменные $X^{\prime}=X / a, Y^{\prime}=Y / a, Z^{\prime}=Z / a^{\prime}$, $j^{\prime}=j \cdot a$. В новые аналоги выражений $(1)-(11)$ величины $a$ и $\gamma$ входят только в виде произведения $a \cdot \gamma=\gamma^{\prime}$.

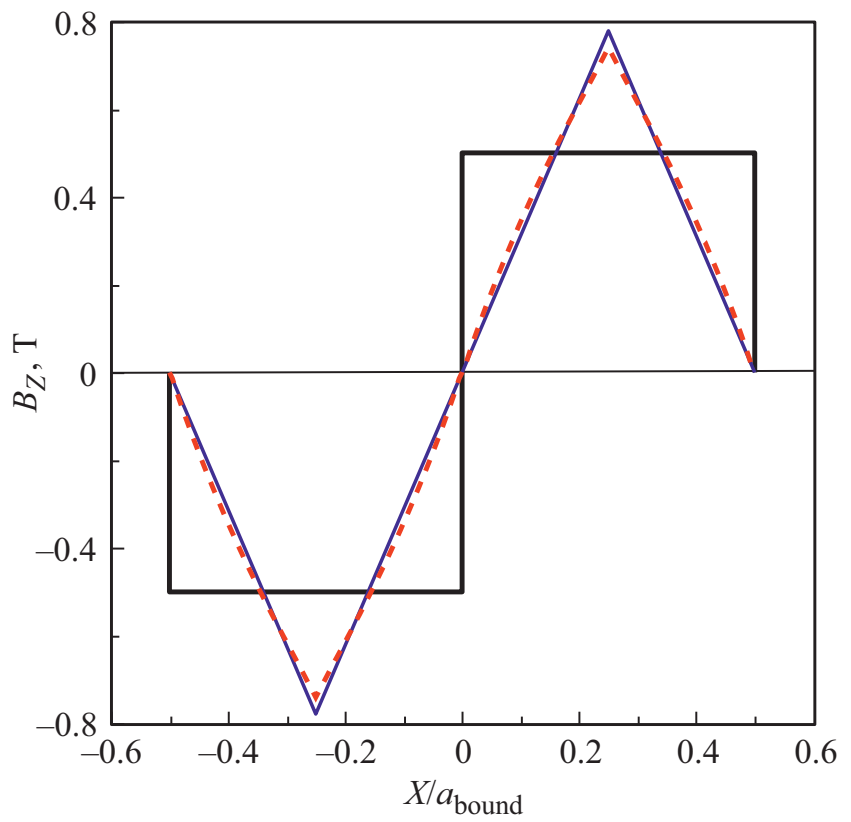

Рис. 5. Один период вертикальной компоненты $B_{Z}$ индукции магнитного поля над поверхностью линейки $(Z=0)$. Черная сплошная линия - магнитная линейка на основе постоянных магнитов. Синяя сплошная линия - магнитная линейка на основе пакетов ВТСП лент (приближение Бина). Красная пунктирная линия - магнитная линейка на основе пакетов ВТСП лент (двухэкспоненциальное приближение).

Решение математической задачи (1)-(11) в новых переменных определяет граничный параметр

$$
\gamma_{\text {bound }}^{\prime}=\gamma(T) \cdot a_{\text {bound }}(T)=f\left(A_{1}, A_{2}, \beta_{1}, \beta_{2}, B_{p m}\right) \text {, }
$$

при котором совпадают первые компоненты рядов Фурье магнитной индукции линеек двух изучаемых типов. Из выражения (12) следует, что в двухэкспоненциальном приближении $a_{\text {bound }}(T=77 \mathrm{~K})=$ $=38 \mathrm{~mm}, a_{\text {bound }}(T=65 \mathrm{~K})=a_{\text {bound }}(T=77 \mathrm{~K}) / 2=19 \mathrm{~mm}$, $a_{\text {bound }}(T=30 \mathrm{~K})=a_{\text {bound }}(T=77 \mathrm{~K}) / 6=6.3 \mathrm{~mm}$. На рис. 5 представлен один период вертикальной компоненты BZ индукции магнитного поля над поверхностью МЛ на основе постоянных магнитов и на основе пакетов ВТСП лент в приближении Бина и в двухэкспоненциальном приближении. На этом рисунке видно, что несмотря на существенное различие величин $a_{\text {bound }}$, получаемых в приближении Бина и в двухэкспоненциальном приближении, в безразмерных координатах $X / a_{\text {bound }}$ эти зависимости практически совпадают. Это совпадение тем более нетривиально, что распределение плотности тока в сверхпроводящей МЛ в двухэкспоненциальном приближении существенно отличается от константы, принятой в модели Бина (смотри рис. 6). На рис. 6 представлены линии уровней безразмерной величины плотности тока $j(X / a, Z / a) / j_{c}(B=0)$. Распределение плотности тока на этом рисунке дано для граничной величины $a=a_{\text {bound }}$. 


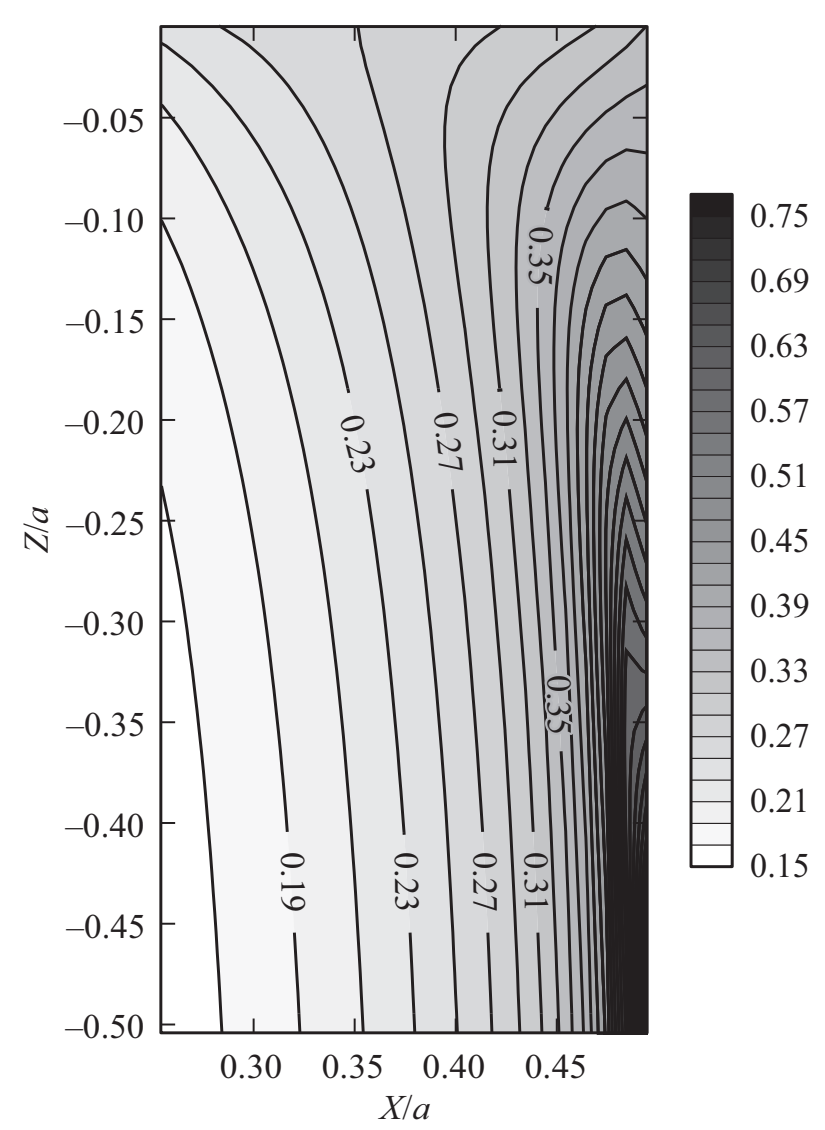

Рис. 6. Линии уровней безразмерной величины плотности тока $j(X / a, Z / a) / j_{c}(B=0)$ в МЛ на основе пакетов ВТСПлент. Изображен верхний фрагмент МЛ $(0.25<X / a<0.5$, $-0.5<Z / a<0)$.

\section{3. Сила взаимодействия верхнего сверхпроводящего слоя с магнитными линейками различного типа}

Определение индуцированных сверхпроводящих токов в верхнем сверхпроводящем слое (ВСС), наведенных полем магнитной линейки возможно в рамках различных подходов. Для описания критических токов в тонкой сверхпроводящей пленке можно использовать подход, основанный на решении уравнений ГинзбургаЛандау [33]. Этот подход позволяет детально определить структуру сверхпроводящих токов, включая структуру отдельного сверхпроводящего вихря, но при решении макроскопических затрат требует слишком высоких затрат компьютерных ресурсов. Более упрощенный подход при описании системы токов оперирует с системой абрикосовских вихрей в магнитном поле, где вихри рассматриваются как отдельные, взаимодействующие друг с другом частицы (смотри, например, [34]), однако и данный подход требует чрезмерных затрат компьютерных ресурсов для расчета токов ВСС, наведенных магнитной линейкой макроскопических размеров. В качестве критерия силового взаимодействия ВСС и
МЛ в представленной работе применяется следующая приближенная модель. Предполагается, что магнитное поле токов, наведенных в ВСС не изменяет намагниченность магнитной линейки. Также предполагается, что ВСС является идеальным диамагнетиком (наведенные в этом слое токи не превышают критических). Идеальный диамагнетизм также подразумевает наличие в ВСС только поверхностных токов, полностью экранирующих внешнее магнитное поле МЛ. Выполнение этого приближения зависит как от расстояния магнитная линейка-верхний сверхпроводящий слой, так и от толщины собственно верхнего сверхпроводящего слоя. Сопоставление силы взаимодействия ВСС проводится как с магнитной линейкой на основе ПМ, так и на основе ВТСП (в рамках двухэкспоненциального приближения). Расчет взаимодействия МЛ-ВСС проводится для температуры $T=77 \mathrm{~K}$ при граничном значении величины периода линеек $a=a_{\text {bound }}=38 \mathrm{~mm}$.

В случае, когда ВСС является идеальным диамагнетиком, нормальная компонента полной индукции магнитного поля на его поверхности равна нулю вне зависимости от расстояния МЛ-ВСС, то есть на поверхности собственная нормальная компонента поля верхнего сверхпроводящего слоя противоположна компоненте магнитной линейки, которая определяется выражениями (1) и (5) для линеек на основе ПМ и ВТСП соответственно. В идеальном диамагнетике наведенные токи являются поверхностными. При этом вектор плотности наведенного магнитного момента ВСС имеет единственную нормальную компоненту $g_{Z}(X, Y)$ (см., например, обзор [17]). Зависимость локального нормального давления $p(X, Y)$ со стороны МЛ с нормальной компонентой $B_{Z}(X, Y, Z)$ на ВCC, нормальная координата поверхности которой равна $Z$, определяется следующим соотношением [15]:

$$
p(X, Y)=g_{Z}(X, Y) \frac{\partial B_{Z}(X, Y, Z)}{\partial Z},
$$

а средняя величина левитационной силы, действующей на единицу поверхности ВСС со стороны МЛ (далее обозначена как Pressure) определяется из (13) следующим образом:

$$
\text { Pressure }=\int_{S} p(X, Y) d X d Y / S,
$$

где $S$ - площадь поверхности ВСС.

Плотности компонент наведенных поверхностных токов ВСС равны соответственно (смотри обзор [17]):

$$
j_{X}(X, Y)=\frac{\partial g_{Z}(X, Y, Z)}{\partial Y}, \quad j_{Y}(X, Y)=\frac{\partial g_{Z}(X, Y, Z)}{\partial X} .
$$

При выбранной геометрии МЛ и ВСС отсутствует зависимость магнитного поля и плотности магнитного момента от координаты $Y$. В этом случае единственной компонентой наведенных поверхностных токов ВСС 
является величина $j_{Y}(X, Y)=j(X)$. На рис. 7 дано сопоставление величины $j(X)$, определяемой выражением (15) и плотности поверхностного тока одной ленты $j_{0}=J_{c} \cdot h=4.155 \cdot 10^{4} \mathrm{~A} / \mathrm{m}$. На этом рисунке представлены плотности тока при $Z=0$ (поверхность ВСС касается поверхности МЛ). Рассматриваются магнитные линейки как на основе ПМ, так и на основе

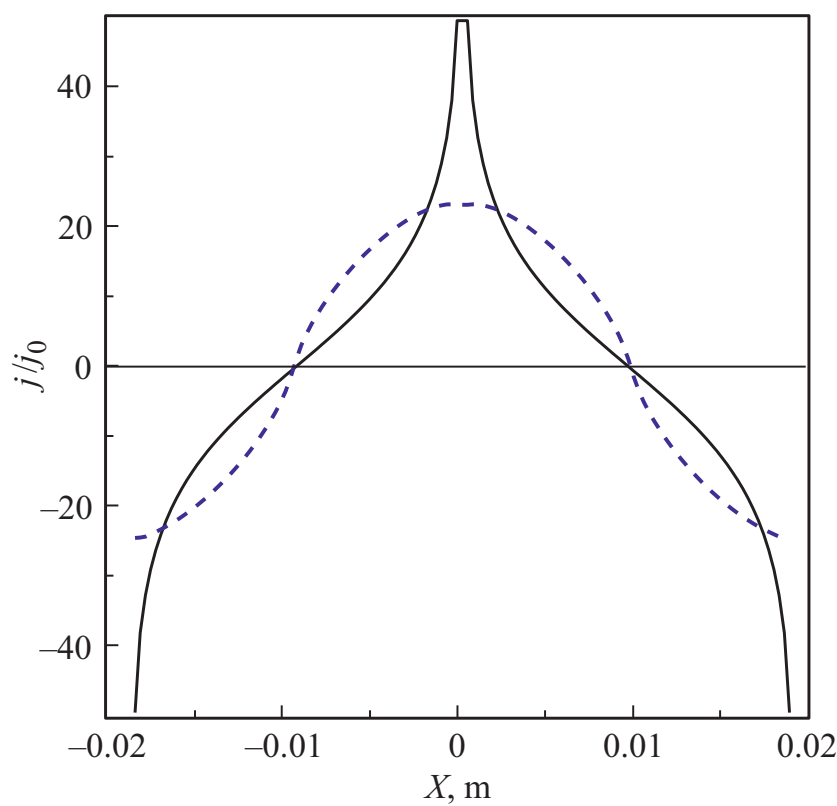

Рис. 7. Зависимость безразмерной величины плотности тока $j(X, Z=0) / j_{0}$ в МЛ на основе ПМ (сплошная черная линия) и на основе пакетов ВТСП-лент (пунктирная синяя линия).

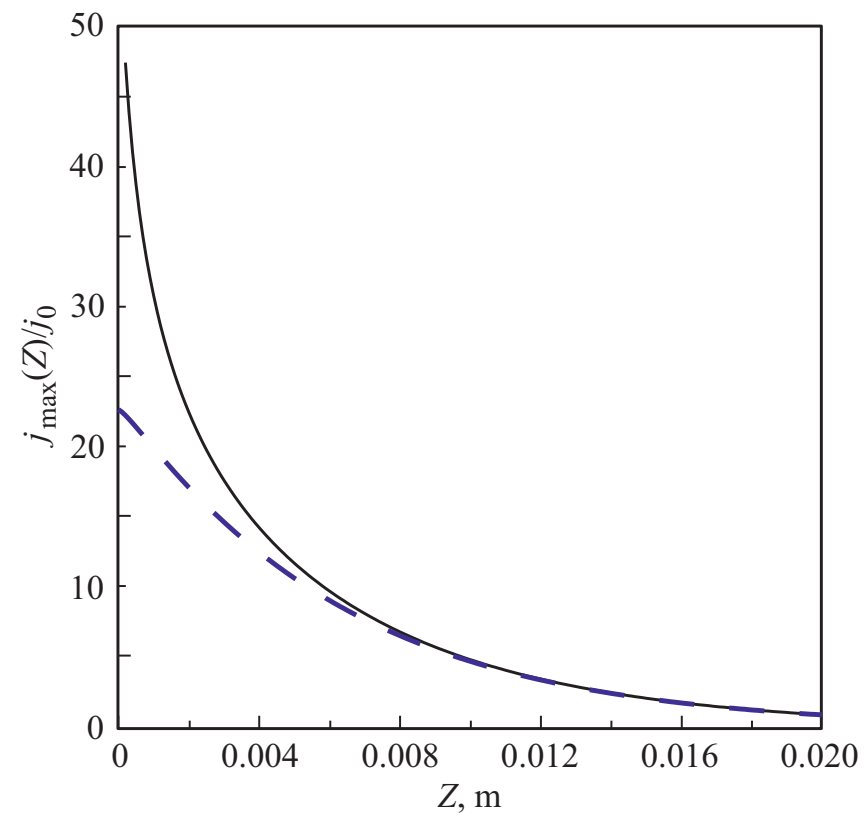

Рис. 8. Зависимость от расстояния $Z$ между МЛ и ВСС максимальной величины плотности тока $j(X=0, Z) / j_{0}$ в МЛ на основе ПМ (сплошная черная линия) и на основе пакетов ВТСП лент (пунктирная синяя линия). Температура $T=77 \mathrm{~K}$, период линеек $a=a_{\text {bound }}=38 \mathrm{~mm}$.

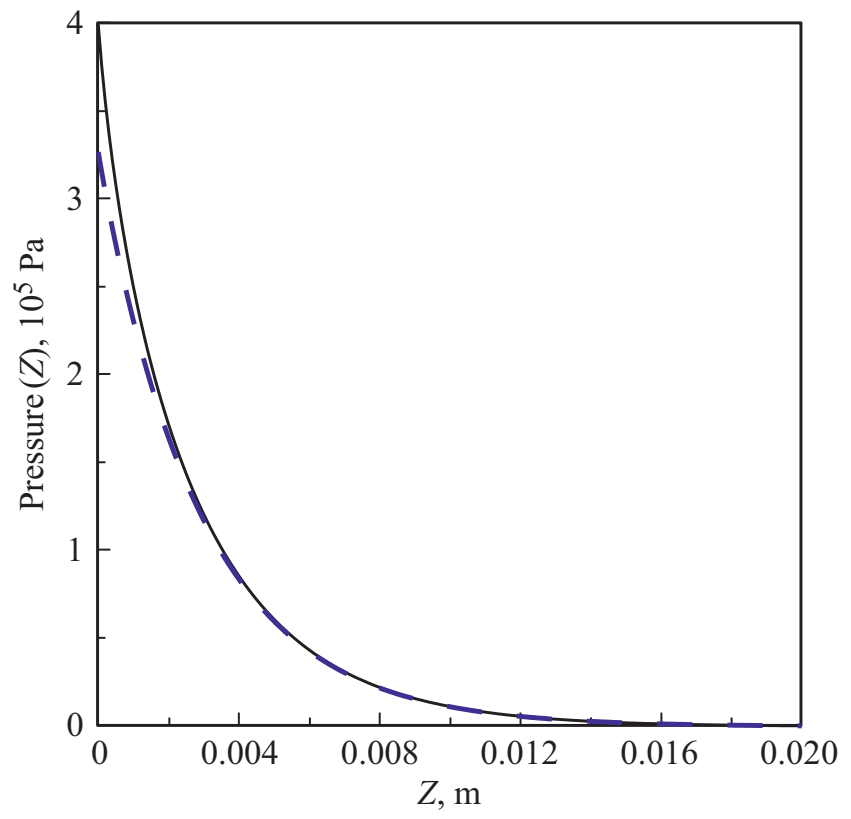

Рис. 9. Зависимость от расстояния $Z$ между МЛ и ВСС среднего значения давления левитационной силы на сверхпроводящий слой со стороны магнитных линеек на основе постоянных магнитов (черная сплошная линия) и пакетов сверхпроводящих лент (синяя пунктирная линия). Температура $T=77 \mathrm{~K}$, период линеек $a=a_{\text {bound }}=38 \mathrm{~mm}$.

ВТСП при температуре $T=77 \mathrm{~K}$ и периоде линеек $a=a_{\text {bound }}=38 \mathrm{~mm}$.

При значительном удалении поверхности ВСС от поверхности МЛ магнитные поля линеек на основе ПМ и ВТСП индуцирующие сверхпроводящие токи в ВСС совпадают (поскольку $a=a_{\text {bound }}$ ). Следовательно, совпадают и наведенные в ВСС-токи. На рис. 8 представлена полученная из выражения (15) зависимость $j_{\max }(Z)-$ максимального значения плотности тока, наведенного в ВСС магнитными линейками различного типа. На рис. 9 представлены полученные из выражения (15) зависимости Pressure(Z). Сопоставление данных рис. 7-9 показывает, что при критическом значении параметра $a=a_{\text {bound }}$ сила левитации при $Z \ll a$, действующая на ВCC со стороны магнитной линейки на основе ПМ незначительно превышает аналогичную величину для линейки на основе ВТСП. Однако линейки на основе ВТСП предпочтительнее, поскольку для полностью диамагнитного поведения ВСС (в приближении Бина) достаточно толщины пакета из $\sim 23$ одиночных лент ВТСП $\left(j_{\max }(Z=0) / j_{0}=23.1\right.$, смотри рис. 7 и 8$)$. Толщина пакета ВСС при этом равна $2.3 \mathrm{~mm}$. Для аналогичного поведения ВСС при использовании МЛ на основе ПМ требуется значительно более толстый пакет лент.

\section{4. Заключение}

Проведенные расчеты показали, что при температуре жидкого азота магнитные линейки на основе пакетов 
ВТСП-лент уступают по эффективности наборам из постоянных магнитов при малых размерах периодов МЛ (до $38 \mathrm{~mm}$ ). При таком периоде для максимального силового взаимодействия между ВТСП-линейкой и пакетом ВТСП-лент, достаточно пакета, содержащего 23 ленты (для рассмотренных лент с плотностью критического тока, равной $\left.j_{0}\right)$. При большей величине периода магнитные линейки на основе пакетов ВТСПлент более эффективны. Эффективность магнитных линеек на основе пакетов ВТСП-лент резко возрастает при даже незначительном понижении температуры (т.е. уменьшается критический размер периода линеек). Так при температуре близкой к температуре жидкого неона магнитные линейки на основе пакетов ВТСП-лент уступают по эффективности наборам из постоянных магнитов при малых размерах периодов МЛ (до $6.3 \mathrm{~mm}$ ), а при большей величине периода магнитные линейки на основе пакетов ВТСП-лент обеспечивают силу магнитной левитации превосходящую силу линейки на основе ПМ.

Полученные результаты имеют не только фундаментальное значение, как характеризующие поведение ВТСП в неоднородных магнитных полях, но также необходимы в прикладной области проектирования различных магнитолевитационных систем.

\section{Финансирование работы}

Исследование выполнено при финансовой поддержке РФФИ, НТУ „Сириус“, ОАО „РЖД“ и Образовательного фонда „Талант и успех“ в рамках научного проекта № 20-38-51012.

\section{Конфликт интересов}

Авторы заявляют, что у них нет конфликта интересов.

\section{Список литературы}

[1] Y. Miyazaki, K. Mizuno, T. Yamashita, M. Ogata, H. Hasegawa, K. Nagashima, S. Mukoyama, T. Matsuoka, K. Nakao, S. Horiuch, T. Maeda, H. Shimizu. Cryogenics 80, 234 (2016).

[2] I. Valiente-Blanco, E. Diez-Jimenez, C. Cristache, M.A. Alvarez-Valenzuela, J.L. Perez-Diaz. Tribology Lett. 6 (2013). DOI: $10.1007 / \mathrm{s} 11249-013-0204-0$

[3] F. Antoncik, M. Lojka, T. Hlasek, V. Bartunek, I. ValienteBlanco, J.L. Perez-Diaz, O. Jankovsky. Supercond. Sci. Technol. (2020). In press. https://doi.org/10.1088/1361-6668/ab6ebe

[4] J.G. Storey, M. Szmigie, F. Robinson, S.C. Wimbush, R.A. Badcock. IEEE Transact. Appl. Supercond. 30, 4, 600706 (2020). DOI: 10.1109/TASC.2020.2982884.

[5] M. Osipov, I. Anishenko, A. Starikovskii, D. Abin, S. Pokrovskii, A. Podlivaev, I. Rudnev. Supercond. Sci. Technol. 34, 035033 (2021). DOI.org/10.1088/1361-6668/abda5a

[6] А.И. Подливаев, И.А. Руднев. ЖТФ 90, 4, 593 (2020). DOI: $10.21883 /$ JTF.2020.04.49082.261-18

[7] J.H. Durrell, A.R. Dennis, J. Jaroszynsk, M.D. Ainslie, K.G.B. Palmer, Y.-H. Shi, A.M. Campbell, J. Hull, M. Strasik,
E.E. Hellstrom. Supercond. Sci. Technol. 27, 8, 082001 (2014).

[8] В.Р. Романовский. ЖТФ 87, 1, 49 (2017).

DOI: 10.21883/JTF.2017.01.44018.1823

[9] В.Р. Романовский. ЖТФ 87, 4, 540 (2017). DOI: 10.21883/JTF.2017.04.44313.1943

[10] K. Liu, W. Yang, G. Ma, L. Queva, T. Gong, C. Ye, X. Li, Z. Luo. Supercond. Sci. Technol. 31, 015013 (2018).

[11] T.P. Yadav, A. Srivastava, G.C. Kaphle. ФTT 63, 2, 249 (2021).

[12] В.В. Деревянко, Т.В. Сухарева, В.А. Финкель. ФТТ 60, 3, 465 (2018).

[13] С.В. Семенов, Д.А. Балаев, М.И. Петров. ФТТ 63, 7, 854 (2021).

[14] С.В. Семенов, Д.А. Балаев. ФТТ 62, 7, 1008 (2020).

[15] Л.Д. Ландау, Е.М. Лифшиц. Теория поля. Наука, М. (1988). $512 \mathrm{c}$.

[16] М.В. Козинцева, А.М. Бишаев, А.А. Буш, М.Б. Гавриков, К.Е. Каменцев, Н.А. Нижельский, В.В. Савельев, А.С. Сигов. ЖТФ 87, 6, 875 (2017). DOI: $10.21883 /$ JTF.2017.06.44509.1911

[17] Ch. Jooss, J. Albrecht, H. Kuhn, S. Leonhardt, H. Kronmuller. Rep. Prog. Phys. 65, 651 (2002).

[18] A.I. Podlivaev, S.V. Pokrovskii, S.V. Veselova, I.V. Anishchenko, I.A. Rudnev. IEEE Transact. Appl. Supercond. 31, 5, 4601505 (2021) DOI: 10.1109/TASC.2021.3065878

[19] E.H. Brandt, M.V. Indenbom. Phys. Rev. B 48, 12893 (1993).

[20] G.P. Mikitik, E.H. Brandt. Phys. Rev. B 62, 6812 (2000).

[21] C.P. Bean. Phys. Rev. Lett. 8, 250 (1962).

[22] C.P. Bean. Rev. Mod. Phys. 36, 31 (1964).

[23] Д.Ф. Алферов, М.Р. Ахметгареев, Д.В. Евсин, И.Ф. Волошин, А.В. Калинов, Л.М. Фишер, Е.В. Цхай. ЖТФ 88, 1 , 28 (2018).

[24] A. Molodyk, S. Samoilenkov, A. Markelov, P. Degtyarenko, S. Lee, V. Petrykin, M. Gaifullin, A. Mankevich, A. Vavilov, B. Sorbom, J. Cheng, S. Garberg, L. Kesler, Z. Hartwig6, S. Gavrilkin, A. Tsvetkov, T. Okada, S. Awaji, D. Abraimov, A. Francis, G. Bradford, D. Larbalestier, C. Senatore, M. Bonura, A.E. Pantoja, S.C. Wimbush, N.M. Strickland, A. Vasiliev. Sci. Rep. 11, 2084 (2021). 10.1038/s41598-021-81559-z

[25] V. Chepikov, N. Mineev, P. Degtyarenko, S. Lee, V. Petrykin, A. Ovcharov, A. Vasiliev, A. Kaul, V. Amelichev, A. Kamenev. Supercond. Sci. Technol. 30, 124001 (2017).

[26] А.И. Подливаев, И.А. Руднев. ФТТ 63, 6, 712 (2021). DOI: 10.21883/FTT.2021.06.50927.028

[27] A.I. Podlivaev, I.A. Rudnev, N.P. Shabanova. Bull. Lebedev Phys. Institute 41, 351 (2014).

[28] Y.B. Kim, C.F. Hempstead, A.R. Strnad. Phys. Rev. Lett. 9, 306 (1962).

[29] Y.B. Kim, C.F. Hempstead, A.R. Strnad. Phys. Rev. 129, 528 (1963).

[30] J.H.P. Watson. J. Appl. Phys. 39, 7, 3406 (1968).

[31] D. Karmakar. Indian J. Phys. 79, 1107 (2005).

[32] A.I. Podlivaev, I.A. Rudnev. Supercond. Sci. Technol. 30, 035021 (2017). DOI.org/10.1088/1361-6668/aa55aa

[33] П.И. Безотосный, С.Ю. Гаврилкин, К.А. Дмитриева, А.Н. Лыков, А.Ю. Цветков. ФТТ 61, 2, 234 (2019).

[34] А.Н. Максимова, В.А. Кашурников, А.Н. Мороз, И.А. Руднев. ФТТ 63, 1, 65 (2021).

Редактор К.В. Емцев 